This document is published in:

IEEE Transactions on Dielectrics and Electrical Insulation.

Vol. 20, No. 2, April 2013. Doi: 10.1109/TDEI.2013.6508772.

(C) 2013 IEEE. Personal use of this material is permitted. Permission from IEEE must be obtained for all other uses, in any current or future media, including reprinting/republishing this material for advertising or promotional purposes, creating new collective works, for resale or redistribution to servers or lists, or reuse of any copyrighted component of this work in other works. 


\title{
Shielding effect of power transformers tanks in the ultra- high-frequency detection of partial discharges
}

\author{
G. Robles, R. Albarracín, J.M. Martínez-Tarifa \\ Carlos III University \\ Electrical Engineering Department \\ Leganés, Madrid, España
}

\begin{abstract}
Partial discharges (PD) measurement is a well-known technique for judgment of the condition of power transformer offline. Phase-resolved PD measurement is a normalized technique for PD tests, but noise, especially by PD-measurements onsite is sometimes difficult to reject, and a post-processing of a pattern is necessary to separate different sources. Higher-frequency-detection devices and processing strategies are useful for PD classification and identification. Recently, ultra-high-frequency (UHF) detection by means of antennas has been shown to be a promising detection system for both offline and online PD-measurements. However, it is necessary to assess whether PD source separation and classification is possible by means of pulse analysis because some additional UHF noise sources are coupled to the detected signals, and it is not clear whether different sources produce different pulses. In this study, the attenuation effect of the metallic tank of a power transformer on the inner PD activity when measured outside the tank was studied. Additionally, experiments were conducted to detect and characterize two different PD sources (internal and external discharges) using two antennas measuring the same phenomenon inside and outside of a transformer. It will also be shown that broadband UHF signals are useful for PD recognition and that a deep study of frequencies below $500 \mathrm{MHz}$ can separate PD occurring inside from those occurring outside when measured with an antenna outside the tank.
\end{abstract}

Index Terms - UHF measurements, partial discharges, shielding, antennas.

\section{INTRODUCTION}

PARTIAL discharge is a phenomenon that may take place at nominal voltage in the weak region of the insulating system. Partial discharge encapsulates ionization processes that occur on a microscopic scale inside dielectric materials, where electric-field exceeds the inception voltage of the specific insulating material (for example, air voids inside solid/liquid materials, sharp electrodes, etc.). However, these processes do not provoke imminent insulation failure; they degrade the insulation bulk where they arise as a consequence of physical and chemical attacks on the solid/liquid material surrounding areas of the PD-source [1], [2].

Oil-paper insulation systems found in power transformers are also affected by this phenomenon [3], [4], and discharges degrade these materials even at rated voltages if PD-source is permanently active during the operation [5]. Thus, the detection of PD activity has become an important qualifying test for these insulation systems, whose evolution with time can help in the predictive maintenance of these important assets of the power grid [6].

Normalized PD measurements have been conducted by means of capacitive-resistive dividers connected in parallel to insulation systems. Phase-resolved-partial-discharge (PRPD) patterns have been used for years as the classical representation of PD activity useful in recognizing PD sources [7]. However, in real insulation systems, several PD sources can be simultaneously active and not all of them are equally damaging to the dielectric material. Moreover, electrical noise is usually present when measuring $\mathrm{PD}$ activity in power transformers (especially onsite); thus the identification of PD sources is a challenge when taking PD measurements. For these reasons, broadband detection by means of highfrequency current transformers (HFCT) and signal processing in high-frequency and very high-frequency (HF/VHF) ranges have been used to detect, separate and classify PD sources [8], [9].

As an alternative to these electrical measurements, noncontact techniques featuring, for example, electro-acoustic detection systems have also been tested. These sensors are able to locate PD sources, measuring the time of flight of acoustic pressure waves propagating through oil. Additionally, these measurements are less sensitive to electrical noise [10], [11]. Despite these advantages, these sensors cannot detect internal PD sites whose acoustic waves must pass through solid insulation systems and are less sensitive than electrical sensors 


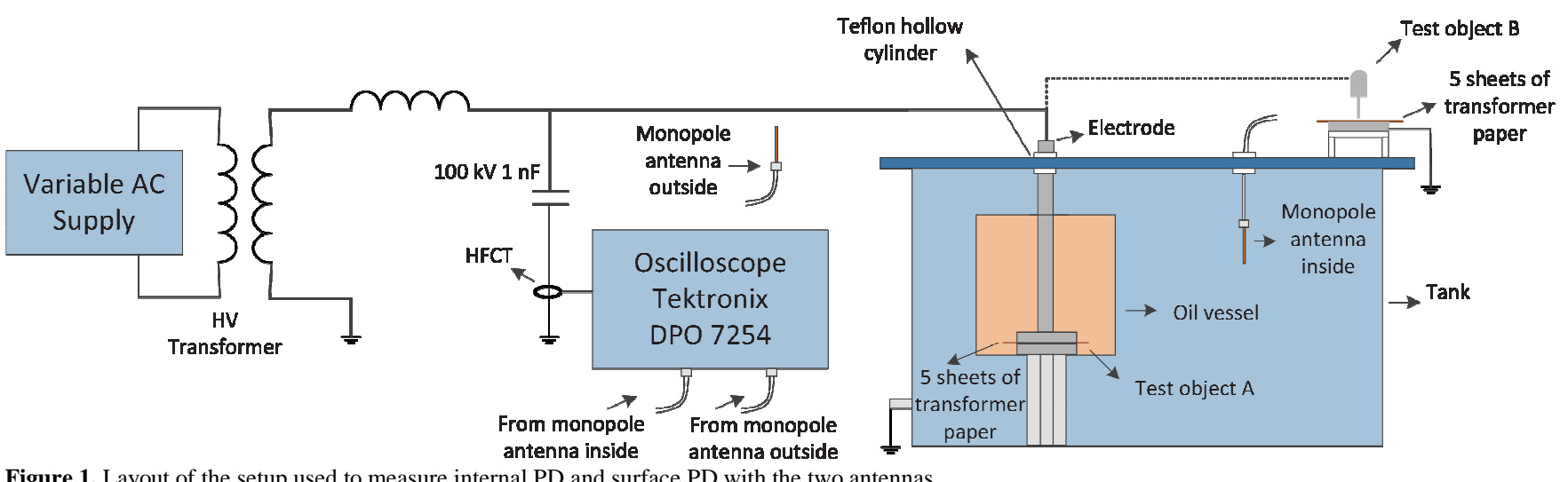

Figure 1. Layout of the setup used to measure internal PD and surface PD with the two antennas.

[10]. As in most acoustic techniques, UHF measurements taken by means of antennas have no galvanic connection to transformer terminals, enabling PD location, and are appropriate for on-line monitoring, exhibiting higher sensitivity than the former [10], [12], [13]. However, different noise sources are present in this frequency range (TV, FM Radio, GSM/GPRS, Wi-Fi, etc.), and PD identification by means of pulse analysis is still a trending research topic [14], [15]. Thus, one of the challenges faced when studying UHF pulses is the possibility of separating different sources because external at transformer bushings and oil-paper internal discharges inside a transformer tank can occur simultaneously. Dielectric windows in transformers, lid layer adjustment and bushing insulation, may allow radiation to pass inside or outside the tank so an antenna could measure both emissions, being internal discharges more difficult to avoid and, thus, more dangerous. Identifying these discharges would greatly improve the predictive maintenance of transformers.

In this paper, the shielding effect of a transformer tank on internal PD pulses created in an oil-paper insulation system is analyzed. Pulse spectra are statistically analyzed with respect to measurements made inside and outside the transformer tank. Internal discharges produced in the oilpaper insulation are measured with an antenna inside the tank and compared to the same discharges measured outside the tank studying their spectra. Surface discharges are measured with the antenna outside the tank, and their spectra are further characterized to identify and separate inner and outer sources.

\section{MEASUREMENT SETUP}

The measurement setup consists of two test objects designed to create stable PD activity, a steel tank to model an oil transformer frame and two monopole antennas. One of the monopoles is located inside the tank at a distance of $0.3 \mathrm{~m}$ from the test object, whereas the other is placed at the same distance but outside the tank. A simplified layout of the setup is shown in Figure 1.

\subsection{TEST OBJECT A}

The first test object is a vessel with two electrodes immersed in transformer oil and separated by 5 sheets of transformer paper. The middle sheet was pierced with a needle to create a small hole measuring $1 \mathrm{~mm}$ in diameter to guarantee that there is a weak spot where internal PD can occur at relatively low voltages. The sheets of paper where impregnated in transformer oil for more than 100 hours to ensure that most of air voids were filled and all discharges were of the same type. This test object is placed inside the tank. One of the electrodes is connected to ground and the other to high voltage through a rod that passes through the tank top lid.

\subsection{TEST OBJECT B}

The second test object is a sharp needle pressing 5 sheets of transformer paper without piercing them. The paper is on a plane electrode connected to ground. This object creates surface discharges perpendicular to the electrical field, as those that can occur in the transformer bushings. Test object $\mathrm{B}$ is located on the tank and is equidistant to the two monopoles.

\subsection{TANK}

The steel tank shown in Figure 2 was built with panels that are $5 \mathrm{~mm}$ thick, as in many high-voltage transformers. This thickness is appropriate to reproduce the same attenuation effects of the electromagnetic radiation emitted from inside. The top lid has two accesses separated by 0.3 $\mathrm{m}$ to connect the high-voltage source to test object $\mathrm{A}$ and one of the monopole antennas. These holes measure $30 \mathrm{~mm}$ in diameter so as to fit a Teflon hollow cylinder that insulates the tank, which is connected to ground, from the high-voltage rod. Finally, a cork layer is used as a mechanical adjustment between the tank and lid, as in many real transformers; thus, the insulating Teflon cylinder for the high-voltage terminals and the cork layer act as dielectric windows for internal UHF signals.

The model of the tank is a simplification of a real transformer tank. The absence of three-phase windings and columns is not critical because we are measuring the attenuation effect and comparing signals measured inside and outside the tank for an invariant inner geometry, which leads to interesting results regarding PD-source separation.

\subsection{MONOPOLE ANTENNAS}

The monopole antennas are made of two $0.1 \mathrm{~m}$ copper wires [16], both soldered to two BNC female connectors connected to coaxial cables measuring $5 \mathrm{~m}$ long. The 

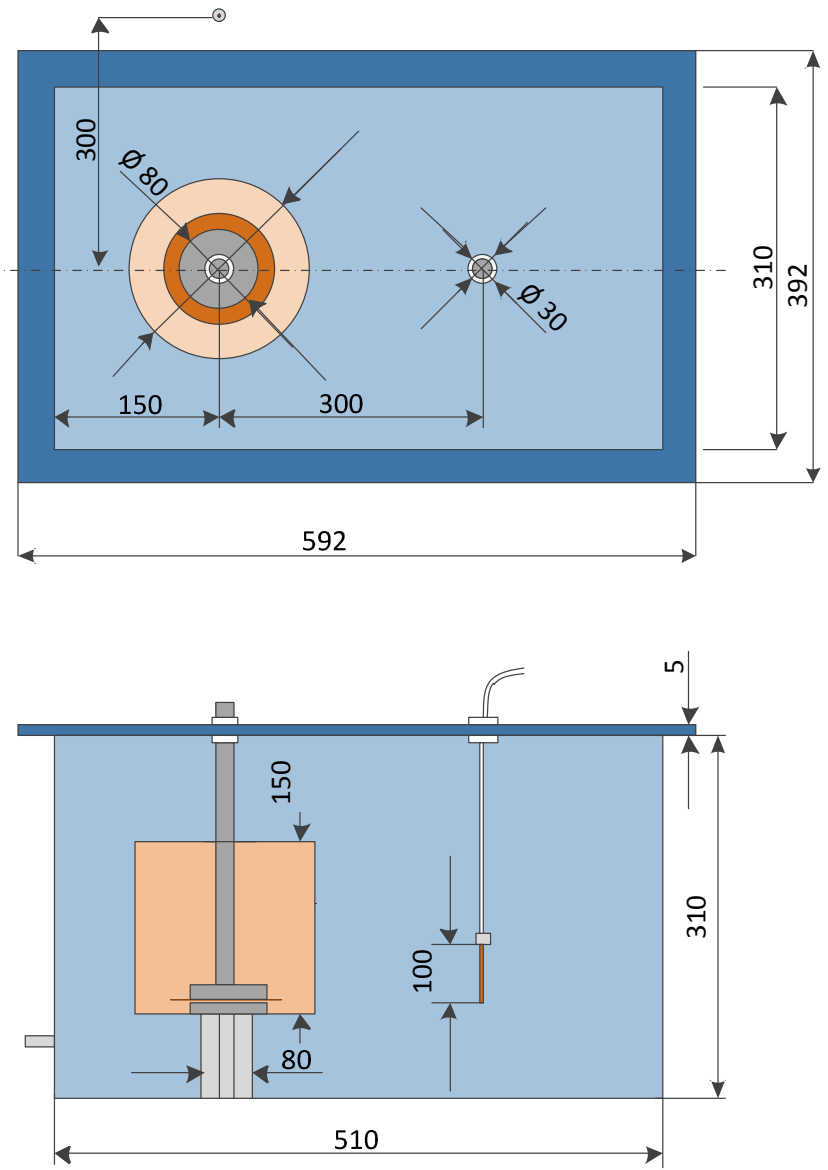

Figure 2. Tank dimensions, in $\mathrm{mm}$.

antennas were connected directly to the $50 \Omega$ input of a Tektronix DPO 7254 oscilloscope.

An antenna was placed inside the tank like in [16]. This antenna is located at a distance of $0.3 \mathrm{~m}$ from test object A, and the other was placed outside the tank at the same distance to have the same time delay for the pulses. To verify that the measurements read by this last antenna were actually an electromagnetic radiation from the PD inside the tank, the antenna was separated $5 \mathrm{~m}$ from the source and a delay of $17 \mathrm{~ns}$ was observed using this experiment setup. When using test object $\mathrm{B}$, the antenna outside the tank is also located $0.3 \mathrm{~m}$ from the object to be consistent with the first experiment.

\subsection{HIGH-VOLTAGE SOURCE AND ACQUISITION SYSTEM}

The high-voltage source is a Schleich BV 702210 transformer with a GLP1-e HV control module. Its output is connected to a $100 \mathrm{kV}, 1 \mathrm{nF}$ coupling capacitor in parallel with the test objects. The PD are also measured with a highfrequency current transformer (HFCT) connected to the ground wire from the capacitor. This HFCT measures the conducted HF signal produced by the partial discharge [17], and it is used to synchronize the radiated UHF signals measured by the antennas when necessary. The output of the HFCT and the antennas are connected to a Tektronix DPO 7254 which acquires the signals at $5 \mathrm{GS} / \mathrm{s}$.
Additionally, a Techimp PDCheck acquisition system was used to detect conventional PRPD patterns from both test objects to corroborate internal and surface PD activity before acquiring UHF signals.

\section{MEASUREMENTS}

\subsection{PARTIAL DISCHARGES IN OIL}

The first set of measurements is aimed at comparing the internal PD in oil measured inside the tank to the same pulses measured outside the tank. Test object $\mathrm{A}$ is connected to the high-voltage transformer, and the voltage is increased until a stable activity of PD is achieved at 2150 $\mathrm{V}$. Then, at least 10 minutes are allowed to elapse to ensure that the activity is stable during the acquisition. The signals in the time domain, acquired in $1 \mu \mathrm{s}$ frames, are as shown in Figure 3. Notice that the UHF signals measured outside the tank are remarkably smaller than inside and not as fast as the pulses obtained with the antenna inside. PD is a stochastic phenomenon whose characteristics depend on environmental conditions, applied voltage, the availability of free electrons and the location of the PD-source regarding the connection to the electrode. Thus, PD pulse analysis requires several measurements at the same applied voltage. To quantify the differences between the signals acquired with the monopoles, a set of 1000 pulses for each antenna was obtained, applying a higher voltage of $5800 \mathrm{~V}$ to test object A to generate larger signals. The FFT with a rectangular window was calculated for all of the pulses; each frequency component was scaled to represent the root mean square and squared to calculate the power of the signal, (1), where $S_{n}$ is the power spectrum at frequency $n$ and $A_{n}$ is the amplitude of the $n-t h$ frequency component.

$$
S_{n}=\left(\frac{A_{n}}{\sqrt{2}}\right)^{2} \mathrm{~V}^{2}
$$

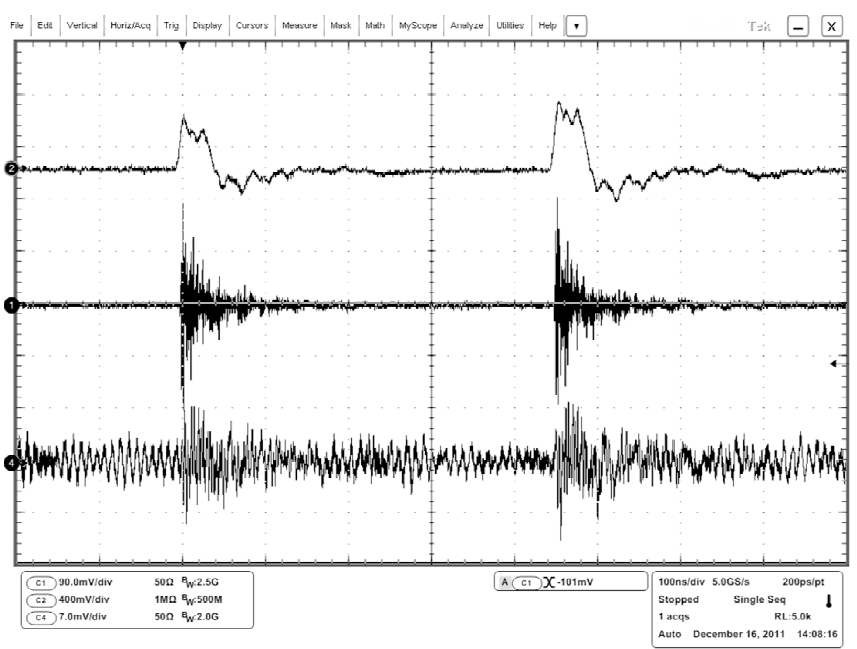

Figure 3. Partial discharge signals in paper impregnated with oil. The upper signal corresponds to the HFCT and has a scale of $400 \mathrm{mV} / \mathrm{div}$. The middle pulses are for the antenna inside the tank and have a scale of 90 $\mathrm{mV} /$ div. Finally, the lower pulses are for the antenna outside the tank and have a scale of $7 \mathrm{mV} / \mathrm{div}$. 


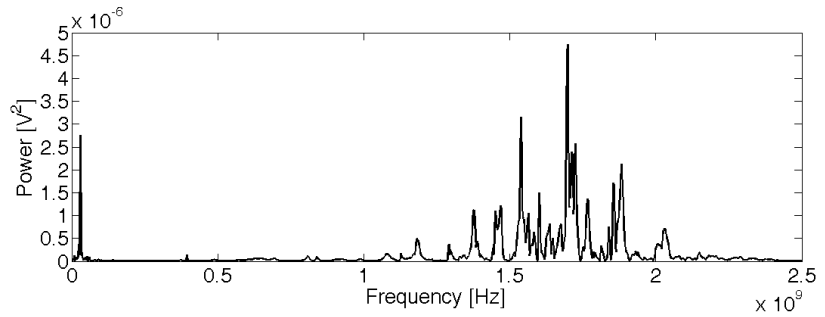

Figure 4. Averaged power spectrum for 1000 signals at $5800 \mathrm{~V}$ measured with the antenna inside the tank for test object A.

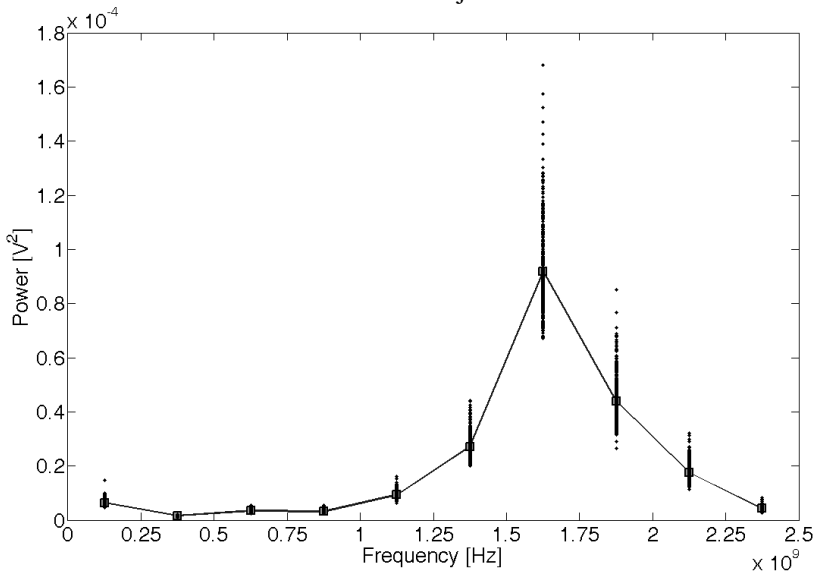

Figure 5. Power per $250 \mathrm{MHz}$ frequency bands of the signals obtained with the antenna inside the tank and average power in those bands for test object A.

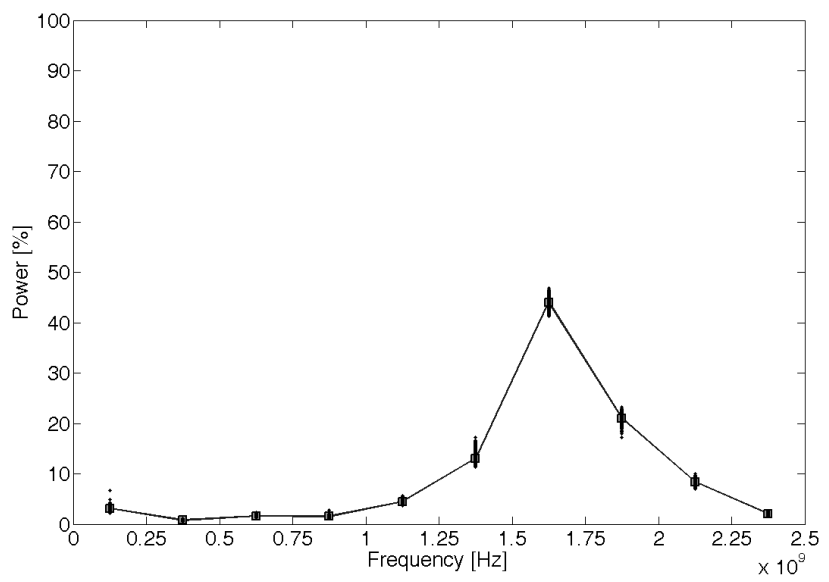

Figure 6. Power percentage per $250 \mathrm{MHz}$ frequency bands of the signals obtained with the antenna inside the tank and average percentage power in those bands for test object A.

Figure 4 shows the averaged power spectrum for 1000 $\mathrm{PD}$ at $5800 \mathrm{~V}$ acquired with the antenna inside the tank.

Figure 5 shows the cumulative power in bands of 250 $\mathrm{MHz}$ for every signal and the average power in those bands calculated using (2), where $f_{L}$ and $f_{H}$ are the lower and upper frequencies of the interval, respectively.

$$
P_{\left[f_{L}, f_{H}\right]}=\sum_{n=f_{L}}^{f_{H}}\left(\frac{A_{n}}{\sqrt{2}}\right)^{2} \mathrm{~V}^{2}
$$

Notice that the dispersion of the power in the bands from $1.5 \mathrm{GHz}$ to $1.75 \mathrm{GHz}$ and from $1.75 \mathrm{GHz}$ to $2 \mathrm{GHz}$ is quite large. This is due to the fact that the signals captured

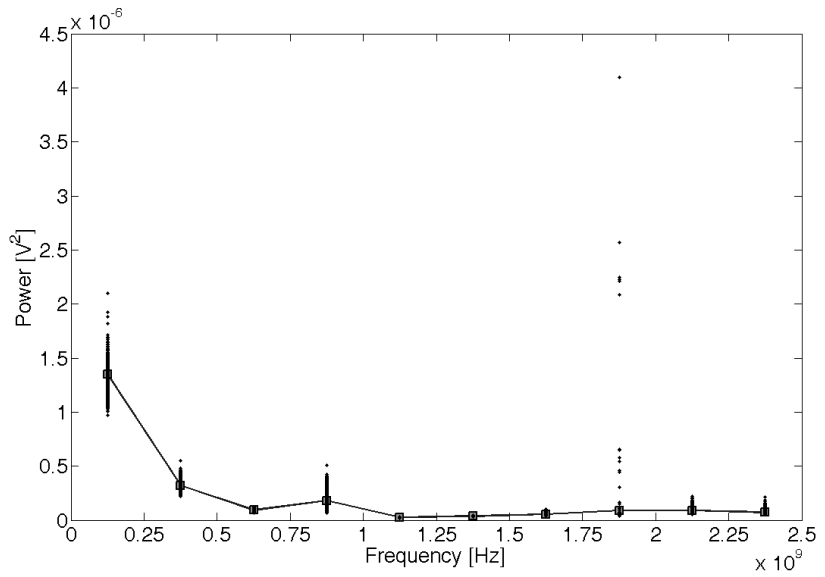

Figure 7. Power per $250 \mathrm{MHz}$ frequency bands of the signals obtained with the antenna outside the tank and average power in those bands for test object A.

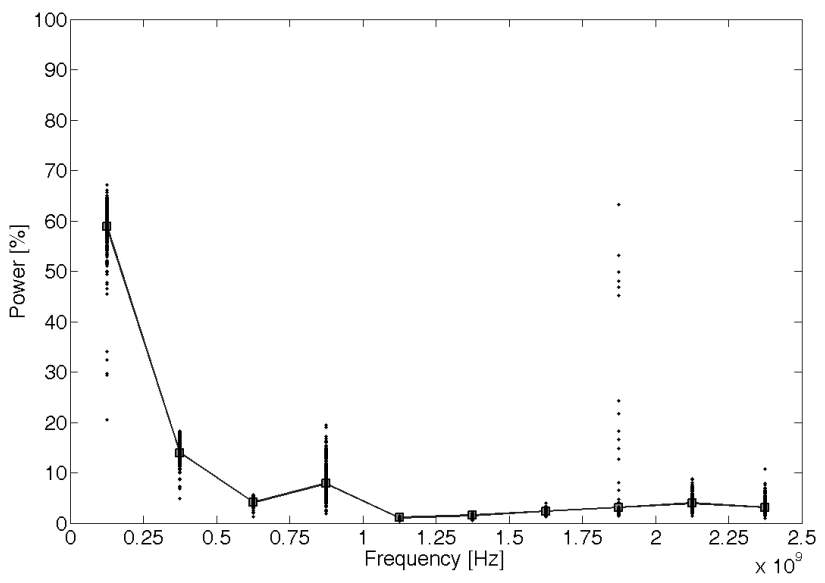

Figure 8. Power percentage per $250 \mathrm{MHz}$ frequency bands of the signals obtained with the antenna outside the tank and average percentage power in those bands for test object A.

by the antenna were very high, exceeding a peak of $5 \mathrm{~V}$ and saturating the oscilloscope, forcing us to set a low trigger to visualize lower signals. This low trigger is less selective, and signals with very disperse powers were acquired as expected from internal PD [1], [3]. Figure 6 shows the percentage of power in bands of $250 \mathrm{MHz}$, demonstrating that almost $80 \%$ of the power in these signals is between $1.25 \mathrm{GHz}$ and $2 \mathrm{GHz}$. This band of frequencies depends on the geometric characteristics of the tank since reflections inside it are directly related to resonances affecting the original PD emission as explained in Section 4.

Figures 7 and 8 illustrate the results obtained with the antenna outside the tank. Notice that the power of the signal is shifted to lower frequencies, which can also be derived from the signals in Figure 3. The tank filters out frequencies in all the studied band since the total average power of the signals outside the tank is below $2 \cdot 10^{-6} \mathrm{~V}^{2}$, while the total average power for the signals inside the tank is near $10^{-4} \mathrm{~V}^{2}$. The band from $1.25 \mathrm{GHz}$ to $2 \mathrm{GHz}$ is now negligible because the reflections are confined inside the tank and do not leak outside. A problem might arise when measuring signals outside the tank because their 


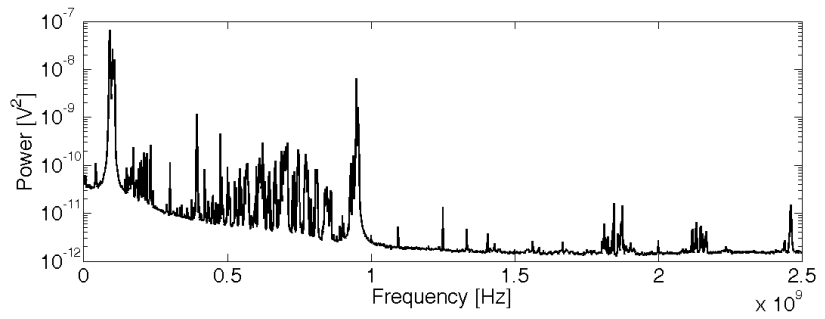

Figure 9. Electromagnetic spectrum outside the tank without PD activity.

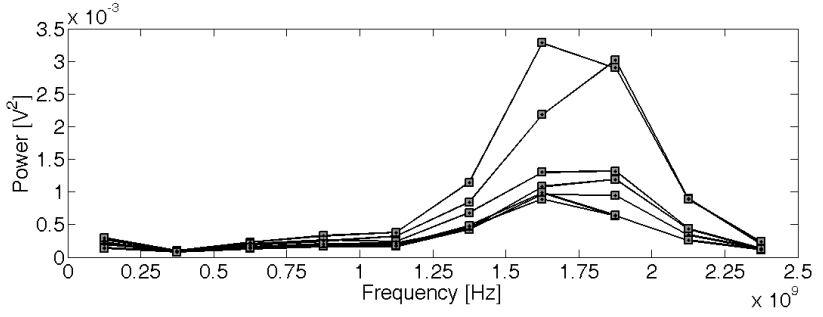

Figure 10. Average power per $250 \mathrm{MHz}$ frequency bands for 7 sets of 1000 signals from the antenna inside the tank for test object $\mathrm{A}$.

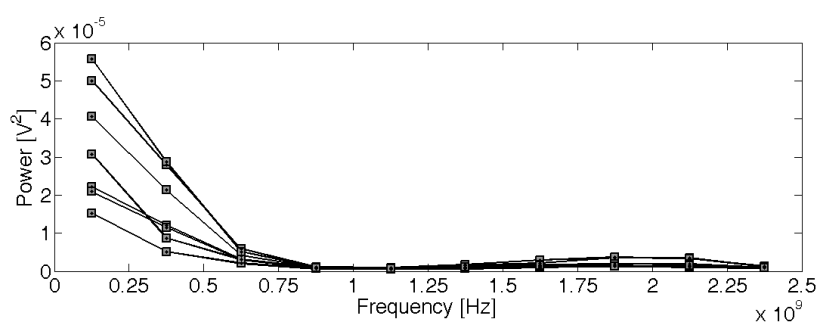

Figure 11. Average power per $250 \mathrm{MHz}$ frequency bands for 7 sets of 1000 signals from the antenna outside the tank for test object A.

higher-energy frequency band is between $0 \mathrm{~Hz}$ to $250 \mathrm{MHz}$, and it is precisely in this band where the electromagnetic spectrum also has more energy because it contains the Citizen Band at $27 \mathrm{MHz}$ and $\mathrm{FM}$ radio frequencies ranging from 88 to $108 \mathrm{MHz}$. Figure 9 represents the electromagnetic spectrum measured by the monopole antenna outside the tank without PD activity. Radio, TV broadcast, GSM at 900 and $1800 \mathrm{MHz}$ and $\mathrm{Wi}-\mathrm{Fi}$ are clearly observed. Notice, however, that the peak power occurs for FM radio and is below $10^{-7} \mathrm{~V}^{2}$; thus, there is a good signal-to-noise ratio for the PD signals acquired with the antenna outside the tank.

Figures 10 and 11 show 7 sets of 1000 signals captured by the antenna inside and outside the tank, respectively. The applied voltages varied from $4500 \mathrm{~V}$ to $5800 \mathrm{~V}$, and the sets of signals were acquired on different days, following the same procedures to ensure the repeatability of the results. These additional experiments confirm the behavior shown in Figures 5 and 7 coinciding even in the order of magnitude of the highest average power band attenuation (around 100 times lower).

\subsection{EXTERNAL DISCHARGES}

The second set of measurements simulates the effect of surface discharges in the transformer bushings, which is why test object B is placed on the tank. The aim of this experiment is to analyze the incidence of surface discharges on an antenna placed outside the tank and to determine if they can obliterate the radiation of the PD occurring inside the tank. Figure 12 shows an example of the surface PD pulses measured with the HFCT and the antennas in test object B. Again, the tank behaves as a resonant cavity and PD signals measured inside it have more amplitude than those measured with the antenna outside

The procedure is the same as that described before; high voltage at $5600 \mathrm{~V}$ is applied to the test object B until PD appears. A waiting time of no less than 10 minutes was allowed to elapse until the activity is constant; then, the acquisition can begin. Figure 13 shows the power per 250 $\mathrm{MHz}$ band for a set of 1000 surface signals acquired with the antenna outside the tank. Notice that the power in the 0$250 \mathrm{MHz}$ band shows orders of magnitude similar to those measured in the case of the PD in oil acquired with the antenna outside. This suggests that, when measuring outside the tank, outer discharges spectra are similar to those of the internal discharges in oil, and they can only be distinguished through further study. The averaged power spectrum has

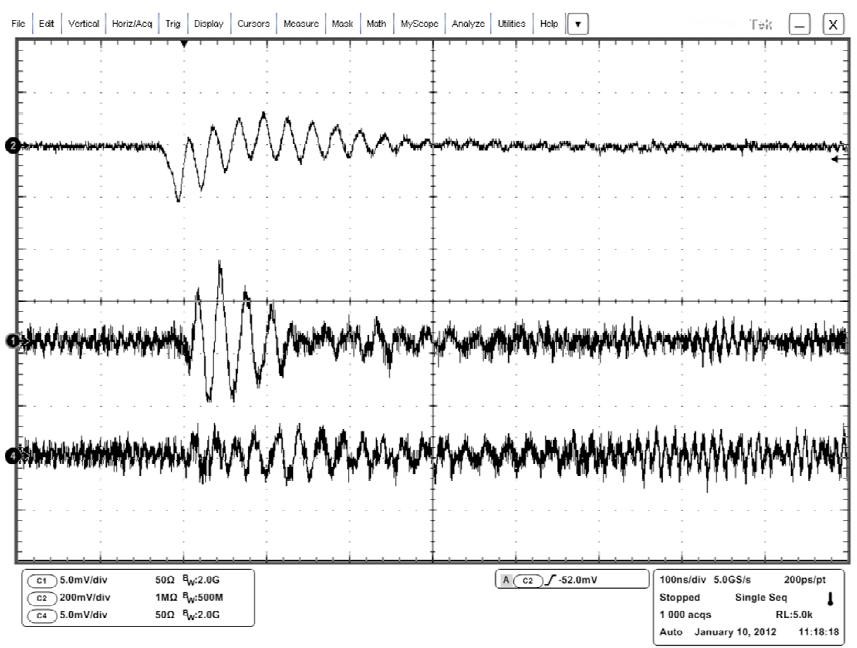

Figure 12. Surface pulses in test object B. The upper signal is the pulse measured with the HFCT ( $200 \mathrm{mV} / \mathrm{div})$. The middle signal is measured with the antenna inside the tank $(5 \mathrm{mV} / \mathrm{div})$, and the lower signal is measured with the antenna outside the tank ( $5 \mathrm{mV} / \mathrm{div})$.

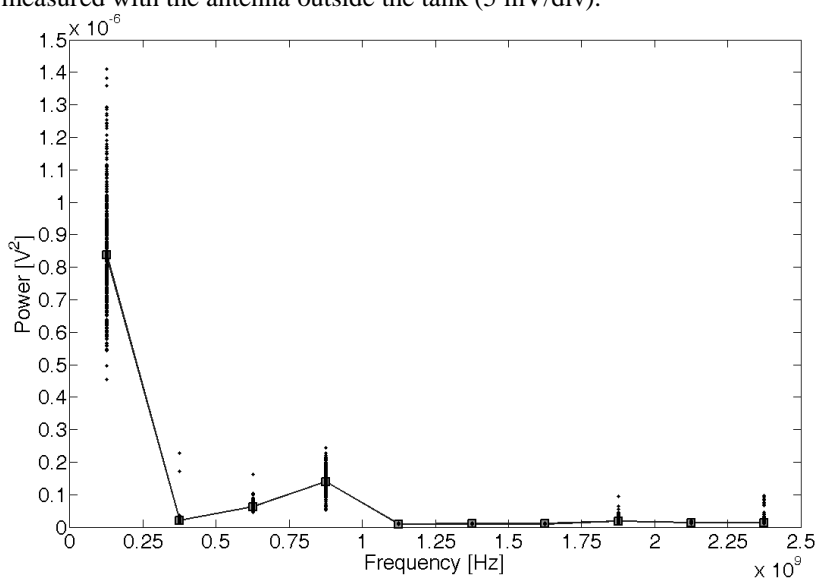

Figure 13. Power per $250 \mathrm{MHz}$ frequency band for 1000 surface signals from the antenna outside the tank and average power in those bands for test object B. 


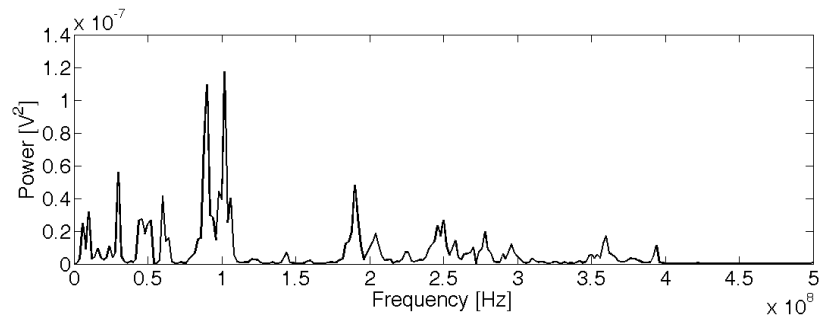

Figure 14. Averaged power spectrum for 1000 signals in test object A at $5800 \mathrm{~V}$ obtained with the antenna outside the tank.

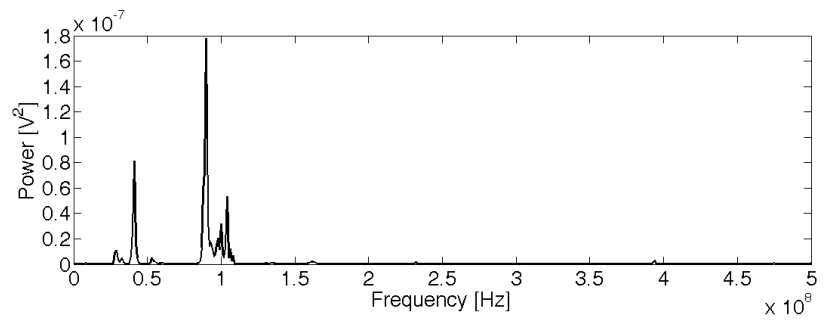

Figure 15. Averaged power spectrum for 1000 signals in test object $B$ at $5600 \mathrm{~V}$ obtained with the antenna outside the tank.

been calculated for two sets of measurements that were performed. The first set consists of 1000 partial-discharge pulses measured with the antenna outside the tank when test object A has a voltage of $5800 \mathrm{~V}$. Figure 14 shows the averaged power spectrum limited to $500 \mathrm{MHz}$. The second set consists of 1000 surface discharges measured with the same antenna when test object $\mathrm{B}$ is at $5600 \mathrm{~V}$; a magnification of the spectra up to $500 \mathrm{MHz}$ is shown in Figure 15. Notice that there is a clear difference between the two power spectra; in the case of surface discharges in test object $\mathrm{B}$, the spectrum is clear and the power is concentrated in the FM-radio band, which is not particularly interesting, and below $50 \mathrm{MHz}$, which is actually due to the power of the surface discharges. In the spectrum of the internal PD measured outside the tank, it is also easy to observe the FM-radio band, and a set of frequencies ranging from $150 \mathrm{MHz}$ to $400 \mathrm{MHz}$ that does not exist in the case of surface discharges. Below $50 \mathrm{MHz}$, the differences are also clear, providing the opportunity to distinguish between the two types of pulses.

\section{DISCUSSION}

Signals inside the tank have spectral components with high power approximately in the band from 1.25 $\mathrm{GHz}$ to $2 \mathrm{GHz}$ as seen in Figures 4, 5 and 6. This broad band of frequencies is no longer appreciable when the measurements are taken without the lid of the tank, so it is easy to infer that they are due to internal reflections. Then, signals acquired with the antenna inside the tank have spectral power in all the measured spectra up to $2.5 \mathrm{GHz}$ but, due to the resonances, the power is concentrated from $1.25 \mathrm{GHz}$ to 2 GHz. When the PD are measured outside the tank, the power in all bands is reduced being this more noticeable in that band because the tank is a resonant chamber and do not allow the leakage of those frequencies. To explain this behavior, the paths followed by the PD emission have to be studied. Figure 16 shows the paths with only one reflection because, as a first approximation, we have considered that the losses due to two reflections would deplete the power of the signal. The Figure 16 shows the direct emission from the PD source to the internal antenna, the reflection on the lid and the reflections on two walls of the tank. The direct emission travels for $30 \mathrm{~cm}$ while the reflections have to travel longer distances, from approximately $43 \mathrm{~cm}$ in the case of reflections on the lid and the closest wall to $51 \mathrm{~cm}$ in the case of the reflection on the furthest wall. This leads to differences in traveled lengths from $13 \mathrm{~cm}$ to $21 \mathrm{~cm}$. Assuming that the transmission medium is air, the differences in times of arrival for the electromagnetic wave would be $0.43 \mathrm{~ns}$ and $0.7 \mathrm{~ns}$, respectively. These times correspond to sinuses with frequencies from $1.4 \mathrm{GHz}$ to $2.3 \mathrm{GHz}$, so a single frequency emission between these frequencies will amount to a constructive interference, doubling the signal received by the antenna. The dispersion throughout this band is explained by the fact that the reflections will not follow exactly these paths because the walls show certain roughness, the $\mathrm{PD}$ can occur in different sites inside the test object, there can be reflections in the electrodes before exiting the test object, etc. that can lead to different lengths in the paths and different constructive interferences. Besides, PD pulses have wideband spectra and emit in many wavelengths that would amount to the dispersion in the band. Additionally, the contrary effect will also occur and there will be destructive interferences in the band from $700 \mathrm{MHz}$ and $1.15 \mathrm{GHz}$ at half the frequencies in the constructive interferences.

It is clear from these results that the tank geometry and the location of the PD source and antenna are critical and will lead to signal amplification or cancellation at different frequencies than those shown here. Moreover, real power transformers will have a magnetic circuit and oil or impregnated paper as transmission medium that would increase the time delays reducing the frequency range affected from resonance. In any case, this study demonstrates that this resonance phenomenon must be taken into account when detecting PD levels by means of antennas inserted through valves inside power transformers.

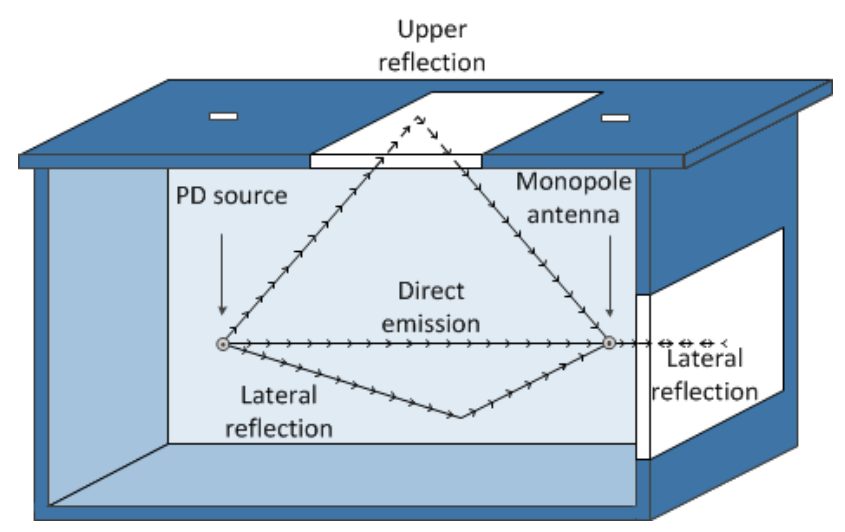

Figure 16. PD reflections in the tank. 


\section{CONCLUSION}

In this paper, PD generated inside a transformer tank have been analyzed using UHF measurements made inside and outside this metallic barrier. It is clear from these results that the steel walls of power transformers lead to the attenuation of the amplitudes of internal PD signals. Resonances at frequencies that strongly depend on the geometry of the tank are found in the measurements done inside. These frequencies do not appear outside the tank due to the fact that it behaves as a resonant chamber in that band. Then, signals measured outside the tank have their spectra biased towards lower frequency ranges. In addition, internal oil-paper PD have been shown to exhibit different spectra for frequencies under approximately $500 \mathrm{MHz}$ than external discharges when measured using external antennas. These results provide useful hints to identify external PD sources in substations that may otherwise be identified as internal PD, leading to inaccurate condition assessment of the oil-paper insulation systems.

\section{ACKNOWLEDGMENT}

This research has been supported by the Spanish Science and Technology Ministry under contract DPI 200914628-C03-02 and by the Madrid Regional Government and Universidad Carlos III de Madrid under contract CCG10-UC3M/DPI-4627.

Tests have been made in the High Voltage Research and Tests Laboratory of Universidad Carlos III de Madrid (LINEALT).

\section{REFERENCES}

[1] L. Niemeyer, "A generalized approach to partial discharge modeling," IEEE Transactions on Dielectrics and Electrical Insulation, vol. 2, pp. $510-528$, aug 1995.

[2] P. Morshuis, "Degradation of solid dielectrics due to internal partial discharge: some thoughts on progress made and where to go now," IEEE Transactions on Dielectrics and Electrical Insulation, vol. 12, pp. 905 - 913, oct. 2005.

[3] Rui-jin Liao; Li-jun Yang; Jian Li; Grzybowski, S.; , "Aging condition assessment of transformer oil-paper insulation model based on partial discharge analysis," IEEE Transactions on Dielectrics and Electrical Insulation, vol.18, no.1, pp.303-311, February 2011.

[4] Okabe, S.; Ueta, G.; Wada, H.; Okubo, H.; , "Partial dischargeinduced degradation characteristics of insulating structure constituting oil-immersed power transformers," IEEE Transactions on Dielectrics and Electrical Insulation, vol.17, no.5, pp.1649-1656, October 2010

[5] IEEE Std C57.104-2008, IEEE Guide for the Interpretation of Gases Generated in Oil-Immersed Transformers, 2009.

[6] F. H. Kreuger, Partial Discharge Detection in High-Voltage Equipment. Londres: Butterworths, 1989.

[7] IEC 60270, High Voltage Test Techniques. Partial Discharge Measurements, 3.0 ed., 2000.

[8] A. Cavallini, G. Montanari, A. Contin, and F. Pulletti, "A new approach to the diagnosis of solid insulation systems based on PD signal inference," IEEE Electrical Insulation Magazine, vol. 19, pp. 22-30, Mar-Apr 2003.

[9] J. M. Martínez-Tarifa, G. Robles, M. V. Rojas-Moreno, and J. SanzFeito, "Partial discharge pulse shape recognition using an inductive loop sensor," Measurement Science and Technology, vol. 21, no. 10 , p. $105706,2010$.

[10] S. M. Markalous, S. Tenbohlen, and K. Feser, "Detection and location of partial discharges in power transformers using acoustic and electromagnetic signals," IEEE Transactions on Dielectrics and Electrical Insulation, vol. 15, Dec 2008.

[11] J. Ramírez-Niño and A. Pascacio, "Acoustic measuring of partial discharge in power transformers," Measurement Science and Technology, vol. 20, no. 11, p. 115108, 2009.

[12] S. Coenen, S. Tenbohlen, S. Markalous, and T. Strehl, "Sensitivity of UHF PD measurements in power transformers," IEEE Transactions on Dielectrics and Electrical Insulation, vol. 15, pp. $1553-1558$, december 2008.

[13] L. Hao, P. L. Lewin, and S. G. Swingler, "Improving detection sensitivity for partial discharge monitoring of high voltage equipment," Measurement Science and Technology, vol. 19, no. 5, p. 055707, 2008.

[14] P. Moore, I. Portugues, and I. Glover, "A nonintrusive partial discharge measurement system based on RF technology," in Power Engineering Society General Meeting, 2003, IEEE, vol. 2, p. 4 vol. 2666, july 2003.

[15] A. Reid, M. Judd, R. Fouracre, B. Stewart, and D. Hepburn, "Simultaneous measurement of partial discharges using IEC 60270 and radio-frequency techniques," IEEE Transactions on Dielectrics and Electrical Insulation, vol. 18, pp. 444 -455, april 2011.

[16] J. López-Roldán, T. Tang, and M. Gaskin, "Optimisation of a sensor for onsite detection of partial discharges in power transformers by the UHF method," IEEE Transactions on Dielectrics and Electrical Insulation, vol. 15, pp. 1634 -1639, december 2008.

[17] G. Robles, J.M.Martínez, M. Rojas, and J.Sanz, "Inductive sensor for measuring high frequency partial discharges within electrical insulation," IEEE Transactions on Instrumentation and Measurement, vol. 58, Nov 2009.

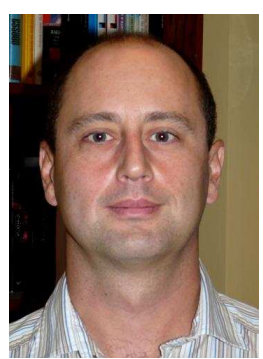

Guillermo Robles (SM'12) was born in Madrid, Spain, in 1969. He received the M.Sc. and Ph.D. degrees in electronic engineering from the Universidad Pontificia de Comillas de Madrid, Madrid, in 1993 and 2002, respectively. He joined the Department of Electrical Engineering, Universidad Carlos III de Madrid (UC3M), Leganes, Madrid in 2002, and he is an Associate Professor in this Department since 2009. He is also with the High-Voltage Research and Tests Laboratory (LINEALT), Universidad Carlos III de Madrid. His research interests include the design of sensors, instrumentation and measurement techniques for high frequency currents, especially due to partial discharges in noisy environments, as well as the study and characterization of magneto-optic sensors based on the Faraday effect for the measurement of currents and the characterization of the behavior of magnetic materials at high frequencies. He has written more than 50 papers in international journals and conferences.

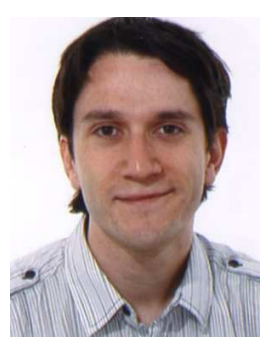

Ricardo Albarracín Sánchez was born in Madrid, Spain, in 1983. He received the degrees of Technical Electrical Engineer and Industrial Electrical Engineer in 2005 and 2008, respectively, and he received his M.Sc. degree in electrical engineering in 2010, from the University Carlos III of Madrid (UC3M), Spain. Since 2008 he is an Assistant Professor in the Department of Electrical Engineering in the UC3M. He is currently a Ph.D. student in electrical engineering at the UC3M. His subject was power grids with distributed generation and integration of renewable sources in power systems, mainly photovoltaic energy sources. In 2011 he joined the High Voltage Research and Tests Laboratory (LINEALT) at UC3M where he is working on insulation systems diagnosis within power cables and electrical machines. 


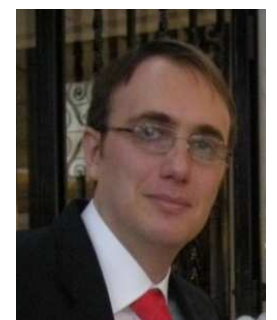

Juan Manuel Martínez-Tarifa (M’ 05) was born in Lorca, Spain in 1975. He received the M.Sc. degree in Electronic Engineering from the Universidad de Granada, Spain in 1999, and the M.Sc. degree in Physics from the Universidad de Granada, Spain in 2000. He received the Ph.D. degree in Electrical Engineering from the Universidad Carlos III de Madrid (UC3M), Spain in 2005. From 2000 to 2012 he has been an Assistant Professor in the Department of Electrical Engineering in UC3M, where he is now Associate Professor. He has been a visiting researcher at Laboratoire de Génie Électrique (Université Paul Sabatier-CNRS, Toulouse, France) and Laboratorio di Ingegneria dei Materiali ed Alte Tensioni (Universitá di Bologna, Italy). $\mathrm{He}$ is currently a Technical Supervisor at the High Voltage Research and Tests Laboratory (LINEALT) at UC3M where he is working on insulation systems diagnosis within power cables and electrical machines. He has published more than 20 articles in International Journals and Conferences. He has joined several research teams to work in more than 10 research projects financed by public and private funds. 